\title{
A hole modulator for InGaN/GaN light-emitting diodes
}

Zi-Hui Zhang, Zabu Kyaw, Wei Liu, Yun Ji, Liancheng Wang, Swee Tiam Tan, Xiao Wei Sun, and Hilmi Volkan Demir

Citation: Appl. Phys. Lett. 106, 063501 (2015);

View online: https://doi.org/10.1063/1.4908118

View Table of Contents: http://aip.scitation.org/toc/apl/106/6

Published by the American Institute of Physics

\section{Articles you may be interested in}

Investigation of $\mathrm{p}$-type depletion doping for InGaN/GaN-based light-emitting diodes Applied Physics Letters 110, 033506 (2017); 10.1063/1.4973743

Advantages of GaN based light-emitting diodes with a p-InGaN hole reservoir layer Applied Physics Letters 100, 141106 (2012); 10.1063/1.3700722

Efficiency droop in InGaN/GaN blue light-emitting diodes: Physical mechanisms and remedies Journal of Applied Physics 114, 071101 (2013); 10.1063/1.4816434

GaN-based light emitting diodes using p-type trench structure for improving internal quantum efficiency Applied Physics Letters 110, 021115 (2017); 10.1063/1.4973995

A hole accelerator for InGaN/GaN light-emitting diodes

Applied Physics Letters 105, 153503 (2014); 10.1063/1.4898588

InGaN/GaN multiple-quantum-well light-emitting diodes with a grading InN composition suppressing the Auger recombination

Applied Physics Letters 105, 033506 (2014); 10.1063/1.4891334

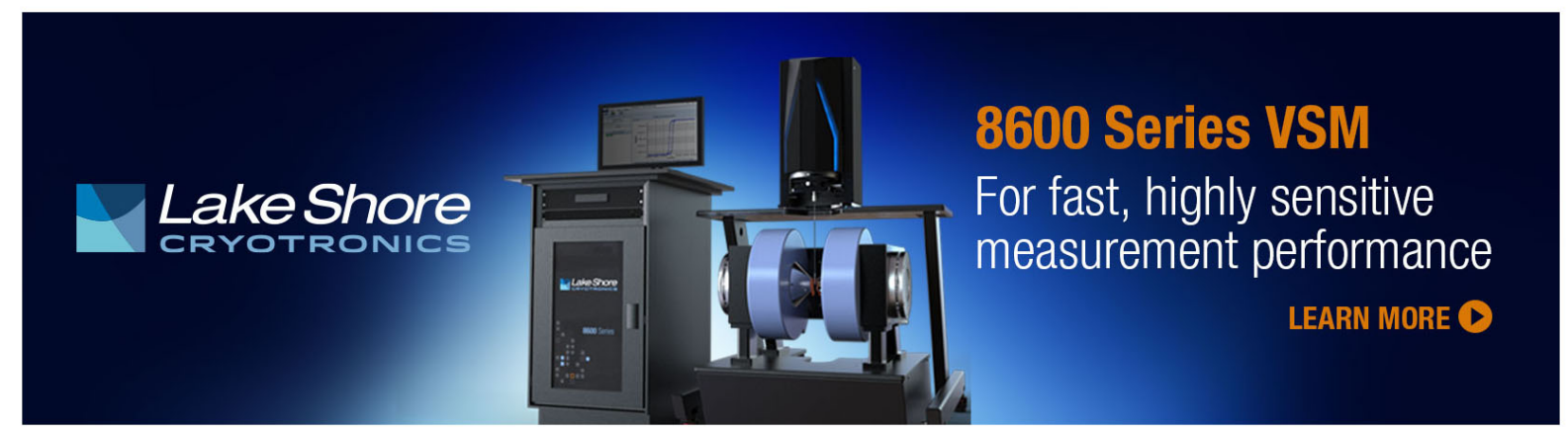




\title{
A hole modulator for InGaN/GaN light-emitting diodes
}

\author{
Zi-Hui Zhang, ${ }^{1}$ Zabu Kyaw, ${ }^{1}$ Wei Liu, ${ }^{1}$ Yun Ji, ${ }^{1}$ Liancheng Wang, ${ }^{1}$ Swee Tiam Tan, ${ }^{1}$ \\ Xiao Wei Sun, ${ }^{1, a)}$ and Hilmi Volkan Demir ${ }^{1,2,3, a)}$ \\ ${ }^{1}$ LUMINOUS! Centre of Excellence for Semiconductor Lighting and Displays, School of Electrical \\ and Electronic Engineering, Nanyang Technological University, 50 Nanyang Avenue, Singapore 639798 \\ ${ }^{2}$ School of Physical and Mathematical Sciences, Nanyang Technological University, 50 Nanyang Avenue, \\ Singapore 639798 \\ ${ }^{3}$ Departement of Electrical and Electronics Engineering, Department of Physics, and UNAM-Institute \\ of Materials Science and Nanotechnology, Bilkent University, TR 06800 Ankara, Turkey
}

(Received 2 November 2014; accepted 2 February 2015; published online 10 February 2015)

The low p-type doping efficiency of the p-GaN layer has severely limited the performance of InGaN/GaN light-emitting diodes (LEDs) due to the ineffective hole injection into the InGaN/GaN multiple quantum well (MQW) active region. The essence of improving the hole injection efficiency is to increase the hole concentration in the p-GaN layer. Therefore, in this work, we have proposed a hole modulator and studied it both theoretically and experimentally. In the hole modulator, the holes in a remote p-type doped layer are depleted by the built-in electric field and stored in the p-GaN layer. By this means, the overall hole concentration in the $\mathrm{p}-\mathrm{GaN}$ layer can be enhanced. Furthermore, the hole modulator is adopted in the InGaN/GaN LEDs, which reduces the effective valance band barrier height for the p-type electron blocking layer from $\sim 332 \mathrm{meV}$ to $\sim 294 \mathrm{meV}$ at $80 \mathrm{~A} / \mathrm{cm}^{2}$ and demonstrates an improved optical performance, thanks to the increased hole concentration in the p-GaN layer and thus the improved hole injection into the MQWs. () 2015 AIP Publishing LLC. [http://dx.doi.org/10.1063/1.4908118]

As the most important candidate in the lighting community, GaN-based light-emitting diodes (LEDs) have been world-widely regarded as one of the effective avenues to save energy and combat the global warming. ${ }^{1,2}$ Though significant efforts have been made to maximize the device performance, it is well known that it is a great challenge to achieve excellent $\mathrm{p}-\mathrm{GaN}$ material suitable for highperformance LEDs. On one hand, the $\mathrm{p}-\mathrm{GaN}$ material features a low hole mobility and the low hole mobility, in turn, reflects a less kinetic energy of holes, which leads to the low hole injection efficiency into the InGaN/GaN multiple quantum wells (MQWs). ${ }^{3}$ Thus, various proposals have been reported in improving the hole transport within the InGaN/ GaN MQWs and enhancing the device efficiency including properly thinning the quantum barriers ${ }^{4}$ and quantum wells, ${ }^{5}$ using Mg-doped quantum barriers, ${ }^{6,7}$ adopting tunneljunction cascaded active region, ${ }^{8}$ as well as applying the InGaN quantum barriers. ${ }^{9}$ On the other hand, the InGaN/ GaN LEDs are also impacted by the very low p-type doping efficiency. Although the p-type doping efficiency can be improved by either the thermal rapid annealing ${ }^{10}$ or the lowenergy electron beam irradiation (LEEBI), ${ }^{11}$ the hole concentration in the $\mathrm{Mg}$ doped $\mathrm{p}-\mathrm{GaN}$ layer is still much less competitive than the electron concentration in the Si-doped $\mathrm{n}$-GaN layer. In this regard, several remedies in increasing the p-type doping efficiency have been demonstrated and proven to be effective in improving the hole concentration to certain extent. One promising approach to increase the hole concentration in the p-GaN layer is to adopt the three-dimensional hole gas (3DHG), ${ }^{12,13}$ well known as the polarization doping.

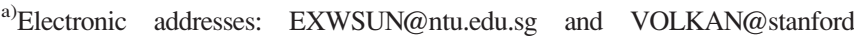
alumni.org
}

Meanwhile, a superlattice-type p-AlGaN/GaN electron blocking layer (EBL) also shows excellent polarization doping effect in increasing the hole concentration. ${ }^{14}$ It has been numerically demonstrated that, by p-type doping the last quantum barrier, the performance of the InGaN/GaN LEDs can be significantly improved. ${ }^{7,15}$ However, the physical mechanism of the improvement is not clearly identified, and the experimental confirmation is needed. In this work, we propose a hole modulator model to explain the physical mechanism of the p-type doped last quantum barrier on the improvement of the InGaN/GaN LED performance. We find that the hole modulator is effective to enhance the hole concentration in the $\mathrm{p}-\mathrm{GaN}$ layer through depleting the remote p-type last quantum barrier region and storing the holes in the $\mathrm{p}$-GaN layer. The effectiveness of the hole modulator in increasing the hole concentration of the $\mathrm{p}-\mathrm{GaN}$ layer is proven through the capacitance-voltage $(\mathrm{C}-\mathrm{V})$ measurement, while the improved device performance is evidenced by measuring the optical output power density and the external quantum efficiency (EQE) for the LED with the proposed design.

Two InGaN/GaN LEDs (LED A and LED B) were grown by a metal-organic chemical vapor deposition (MOCVD) system. The epi-growth was initiated on the c-plane sapphire substrates. We first grew a $20 \mathrm{~nm}$ thick $\mathrm{GaN}$ buffer layer followed by a $4 \mu \mathrm{m}$ thick unintentionally doped n-type $\mathrm{GaN}$ (u-GaN) layer. Then, the Si-doped n-type $\mathrm{GaN}(\mathrm{n}-\mathrm{GaN})$ layer was grown on the u-GaN template, with the thickness and the doping concentration of $2 \mu \mathrm{m}$ and $5 \times 10^{18} \mathrm{~cm}^{-3}$, respectively. Next, we grew six-pair $\operatorname{In}_{0.15} \mathrm{Ga}_{0.85} \mathrm{~N} / \mathrm{GaN}$ MQWs, of which the quantum well and quantum barrier thickness is $3 \mathrm{~nm}$ and $12 \mathrm{~nm}$, respectively. For LED A, all the quantum barriers are u-GaN layers, while the quantum barriers for LED B are also $\mathrm{u}-\mathrm{GaN}$ layers except the last quantum barrier that is adjacent 
to the $25 \mathrm{~nm}$ thick p-type $\mathrm{Al}_{0.20} \mathrm{Ga}_{0.80} \mathrm{~N}$ EBL. The last quantum barrier for LED B is half-doped by $\mathrm{Mg}$ dopants, i.e., $\mathrm{u}-\mathrm{GaN}(6 \mathrm{~nm}) / \mathrm{p}-\mathrm{GaN}(6 \mathrm{~nm})$ along the c-orientation, and the $\mathrm{Mg} / \mathrm{Ga}$ molar ratio for the $\mathrm{Mg}$ doped region in the last quantum barrier is about $0.95 \times 10^{-2}$. The molar ratio of $\mathrm{Mg} /[\mathrm{Al}$ $+\mathrm{Ga}$ ] for the p-type $\mathrm{Al}_{0.20} \mathrm{Ga}_{0.80} \mathrm{~N}$ EBL is $\sim 2.08 \times 10^{-2}$. We then grew the $\mathrm{p}-\mathrm{GaN}$ layer for both LEDs $\mathrm{A}$ and $\mathrm{B}$ acting as the hole injection layer, and the thickness of the $\mathrm{p}-\mathrm{GaN}$ layer is set to $\sim 110 \mathrm{~nm}$ during the epi-growth. The $\mathrm{Mg} / \mathrm{Ga}$ molar ratio for the $\mathrm{p}$-GaN layer is set to $\sim 1.83 \times 10^{-2}$. We finally cover the p-GaN layer with a $\sim 20 \mathrm{~nm}$ thick heavily $\mathrm{Mg}$ doped $\mathrm{GaN}\left(\mathrm{p}^{+}-\mathrm{GaN}\right)$ working as the ohmic contact layer. The molar ratio between $\mathrm{Mg}$ and $\mathrm{Ga}$ precursors is set to $\sim 5.49 \times 10^{-2}$. The in-situ thermal annealing $\left(600 \mathrm{~s}\right.$ at $\left.720^{\circ} \mathrm{C}\right)$ in the $\mathrm{N}_{2}$ ambient was also conducted for the grown LED samples to activate the $\mathrm{Mg}$ dopants in the p-type layers.

The numerical computations for the aforementioned LEDs were performed by APSYS simulation package with physical models and parameters properly set by users. ${ }^{16}$ Important parameters such as the energy band offset ratio of the InGaN/GaN MQWs, the energy band offset ratio of the $\mathrm{GaN} / \mathrm{AlGaN}$ heterojunction, Auger recombination coefficient, and Shockley-Read-Hall (SRH) recombination coefficient can be found in our previously published works. ${ }^{3,4,6,9,13,16,17}$ Here, it is worth mentioning that the polarization effect is considered in our models. We set a $40 \%$ polarization level, such that $60 \%$ of the theoretical polarization charges are released due to the strain relaxation by generating dislocations when compared to the semiconductor epi-layers with perfect crystal quality. ${ }^{18}$ Other parameters on nitrogen-contained compound semiconductors can be found elsewhere. ${ }^{19}$

In order to understand the process of hole injection from the p-GaN layer to the InGaN quantum wells, we first numerically reproduce the energy band diagram for the LED with the $\mathrm{u}-\mathrm{GaN}$ as the quantum barriers (Fig. 1) when the device is forward biased at $80 \mathrm{~A} / \mathrm{cm}^{2}$. Clearly we can see that, when the holes arrive at the interface between the p-type EBL and the last quantum barrier, the holes initially possess a high potential energy. Then, the holes will experience a potential energy decrease when travelling in the last quantum barrier before finally entering the last quantum well. Obviously, the last quantum barrier has little blocking effect

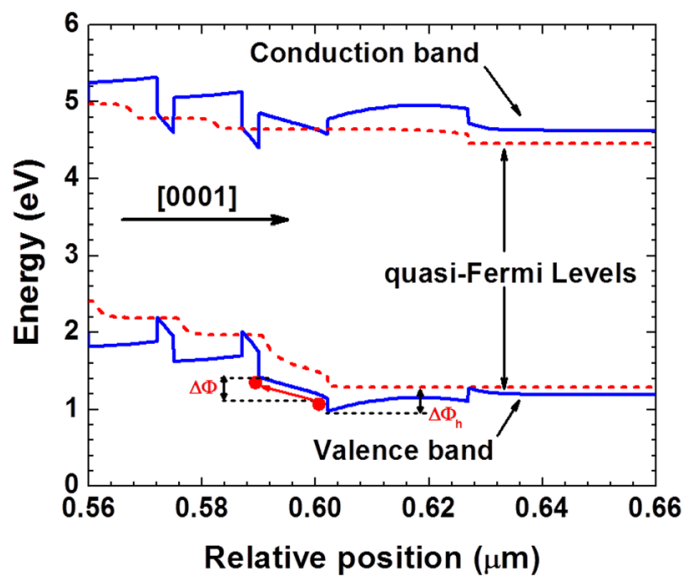

FIG. 1. Energy band diagram for a typical InGaN/GaN MQWs, p-EBL, and p-GaN at $80 \mathrm{~A} / \mathrm{cm}^{2}$. on the hole injection. On the other hand, $\Delta \Phi$ is $\sim 273 \mathrm{meV}$ for LED A and $\sim 271 \mathrm{meV}$ for LED B at $80 \mathrm{~A} / \mathrm{cm}^{2}$, respectively, and thus, such a small $\Delta \Phi$ variance of $\sim 2 \mathrm{meV}$ is less likely to cause the different hole injection efficiency. However, the hole injection efficiency is strongly affected by the $\Delta \Phi_{\mathrm{h}}$, which represents the effective valance band barrier height for the p-EBL. Our calculations illustrate that $\Delta \Phi_{\mathrm{h}}$ is $\sim 332 \mathrm{meV}$ for LED A and $\sim 294 \mathrm{meV}$ for LED B at $80 \mathrm{~A} / \mathrm{cm}^{2}$. Nevertheless, it is not straightforward to envision how p-type doping the last quantum barrier affects the valance band barrier height for the p-EBL and helps to increase the hole injection in the previous reports. ${ }^{7,15}$ The explanation on the origin of the enhanced hole injection into the InGaN/GaN MQW and the improved device performance by the p-type doped last quantum barrier is lacking at present.

By an in-depth analysis, we found that the origin of the enhanced hole injection for the InGaN/GaN LED with the p-type doped last quantum barrier can be interpreted by the concept of the hole modulator, as schematically shown in Fig. 2(a), in which the holes provided by the p-type doped last quantum barrier are depleted by the built-in electric field and then finally stored in the p-GaN layer. As a result, the overall hole concentration in the $\mathrm{p}-\mathrm{GaN}$ layer will be significantly increased. When the device is biased [see Fig. 2(b)], the enhanced hole concentration in the p-GaN layer is very helpful to reduce the effective valance band barrier height of the p-EBL and promote the hole injection into the InGaN/ GaN MQW active region. ${ }^{17}$

In order to precisely probe the effectiveness of the hole modulator in increasing the hole concentration of the $\mathrm{p}-\mathrm{GaN}$ layer, we numerically calculate the energy bands and the hole profiles for LEDs $\mathrm{A}$ and $\mathrm{B}$ at the equilibrium state [Fig. 3(a)]. The energy bands show that the depletion regions for both LED devices at the equilibrium state mostly lie in the last quantum barrier and the p-type EBL regions, and this is due to the big contrast of the doping concentrations for the $\mathrm{n}-\mathrm{GaN}$ and $\mathrm{p}-\mathrm{GaN}$ layers. The last quantum barrier is partially doped by $\mathrm{Mg}$ dopants for LED B, and the holes supplied by the last quantum barrier are also depleted. These holes are stored in the p-GaN layer, which are also calculated and illustrated in Fig. 3(a). We can see that the holes in the vicinity of the p-type EBL for LED B are distributed in a 2D
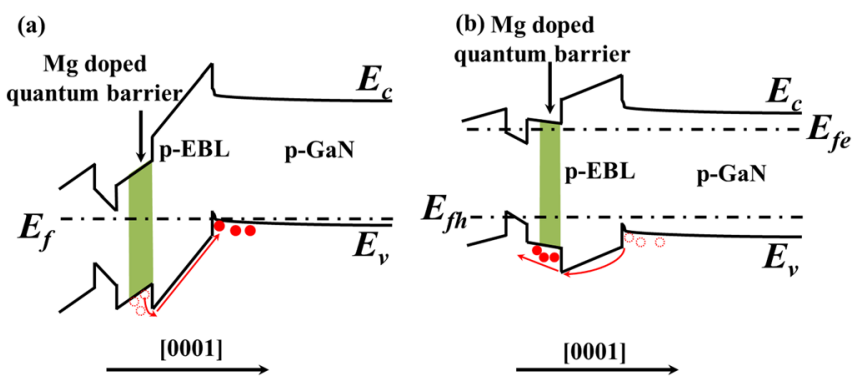

FIG. 2. Schematic energy band diagrams for InGaN/GaN MQWs, p-EBL, and p-GaN: (a) Under no bias, the last quantum barrier is partially p-type doped (indicated in the shadow region). Holes are generated by the remotely p-type doped last quantum barrier. Holes are then depleted by the built-in electric field and finally stored in the p-GaN layer, (b) under bias, the holes will be then injected into the MQWs. $E_{c}, E_{v}, E_{f}, E_{f e}$, and $E_{f h}$ denote the conduction band, valance band, Fermi level, quasi-Fermi levels for electrons, and holes, respectively. 

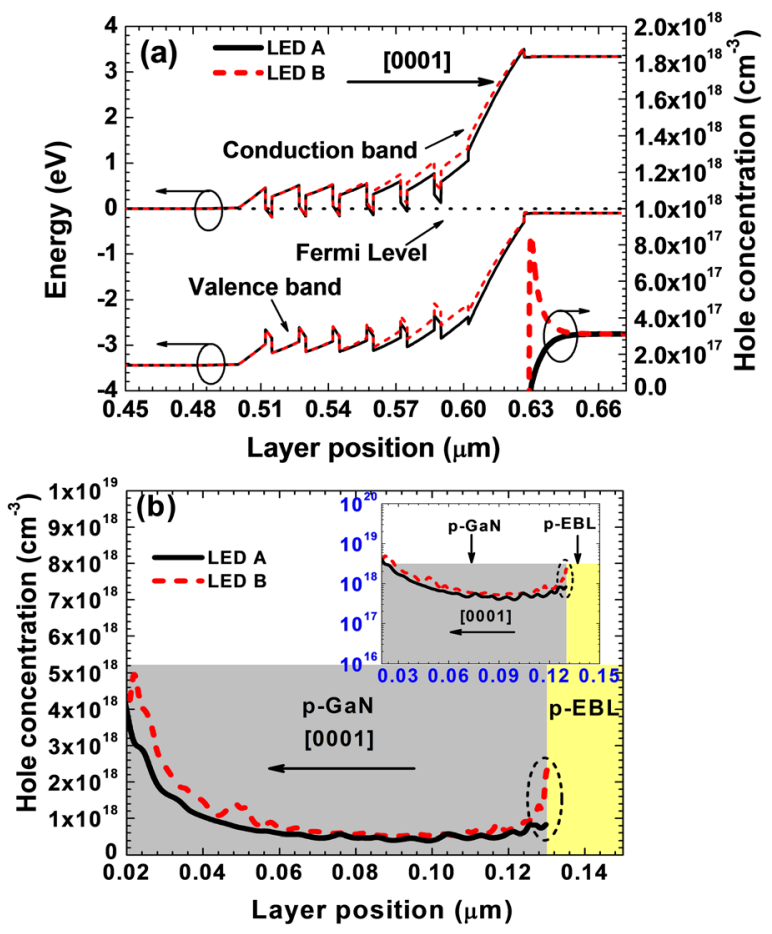

FIG. 3. (a) Calculated energy bands and hole profiles at the equilibrium state and (b) experimentally measured hole profiles within the p-GaN layer for LEDs A and B, respectively. Inset in (b) presents the hole profiles in semi$\log$ scale.

manner, which is due to the polarization induced negative charges at the interface of the p-type EBL and the p-GaN layer. ${ }^{13}$ Nevertheless, the hole concentration in the $\mathrm{p}-\mathrm{GaN}$ layer for LED B is significantly increased compared to that for LED A.

Besides the numerical investigations, we also experimentally characterize the hole profiles within the $\mathrm{p}-\mathrm{GaN}$ layers for LEDs $\mathrm{A}$ and $\mathrm{B}$, respectively, by the capacitance-voltage (C-V) measurement. The $\mathrm{C}-\mathrm{V}$ measurement system used in this work is an 802B MDC three-function mercury probe, which is operated at an AC frequency of $100 \mathrm{kHz}$. The capacitance value for the tested sample is recorded by the circular mercury probe, the area of which is $4.582 \times 10^{-3} \mathrm{~cm}^{-2}$. Details on measuring the carrier concentration by the $\mathrm{C}-\mathrm{V}$ method can be found elsewhere. ${ }^{13,20}$ Fig. 3(b) shows the measured hole concentration profiles in the $\mathrm{p}-\mathrm{GaN}$ layers for LEDs A and B, respectively. We can see that the hole concentration in the spatial range between $0.05 \mu \mathrm{m}$ and $0.12 \mu \mathrm{m}$ for both LEDs A and B is around $4 \times 10^{17} \mathrm{~cm}^{-3}$. However, the hole concentration is higher than $4 \times 10^{17} \mathrm{~cm}^{-3}$ when the layer position is smaller than $0.05 \mu \mathrm{m}$, and this is due to the $\mathrm{Mg}$ diffusion into the $\mathrm{p}-\mathrm{GaN}$ layer from the $\mathrm{p}^{+}-\mathrm{GaN}$ layer, which therefore increases the $\mathrm{Mg}$ doping concentration. It is interesting to see an much higher hole concentration for LED $\mathrm{B}$ than that for LED A at the layer position of $0.13 \mu \mathrm{m}$ (the interface of the p-GaN layer and the p-type EBL) in Fig. 3(b), where the hole concentration for LED B is $\sim 2 \times 10^{18} \mathrm{~cm}^{-3}$. Compared to LED A, the increased hole concentration at the layer position of $0.13 \mu \mathrm{m}$ for LED B is contributed by the partially p-type doped last quantum barrier in LED B. The partially p-type doped last barrier for LED B is depleted by the built-in electric field, and the holes are then stored in the p-
GaN layer. Additionally, being consistent with the numerical hole distribution for LED B in Fig. 3(a), the 2D manner of the hole profile at the interface of the p-GaN layer and the p-type EBL is also experimentally observed in Fig. 3(b), and this is due to the carrier realignment by the polarization induced negative charges at the interface of the $\mathrm{p}-\mathrm{GaN}$ layer and the $\mathrm{p}$ type EBL. ${ }^{13}$ An increased hole concentration in the $\mathrm{p}-\mathrm{GaN}$ layer for LED B can then remarkably improve the hole injection efficiency. ${ }^{17}$

It is noted that, although the numerically calculated hole profiles agree well with the experimentally measured hole profiles [refer to Figs. 3(a) and 3(b)], we still can see the slight discrepancies in absolute values of hole concentration between Figs. 3(a) and 3(b), such as the hole concentration at the interface of the p-GaN layer and the p-type EBL. The discrepancy is, on one hand, caused by the difficulty in precisely probing the ionization ratio of the $\mathrm{Mg}$ dopants in the p-type epi-layers. In addition, the Mg diffusion is strongly affected by the epi-growth conditions, and these parameters in our simulation models cannot be accurately set. On the other hand, the polarization level is also hard to be precisely modeled due to the dependence on the growth conditions. However, the slight discrepancies will not change the conclusion of this work.

To further support that the hole modulator is helpful in increasing the hole injection into the InGaN/GaN MQWs, we calculate the hole concentration profiles in the p-EBL/ p-GaN interface [Fig. 4(a)] and across the active regions [Fig. 4(b)] for LEDs A and B at $80 \mathrm{~A} / \mathrm{cm}^{2}$. Fig. 4(a) shows a higher hole concentration at the $\mathrm{p}-\mathrm{EBL} / \mathrm{p}-\mathrm{GaN}$ interface for LED B than for LED A, and this is consistent with results of Figs. 3(a) and 3(b). The increased hole concentration of the $\mathrm{p}-\mathrm{GaN}$ layer, according to the report by Ref. 17, well explains the reduced $\Delta \Phi_{\mathrm{h}}$ in Fig. 1 for holes, ${ }^{17}$ which is $\sim 332 \mathrm{meV}$ for LED A and $\sim 294 \mathrm{meV}$ for LED B at $80 \mathrm{~A} / \mathrm{cm}^{2}$. In addition, Fig. 4(b) indicates a much higher hole concentration in the quantum wells for LED B compared to LED A. The increased hole concentration in the quantum wells is ascribed to the enhanced hole injection promoted by the increased hole concentration in the p-GaN layer for LED B. The increased hole concentration in the active regions is also helpful to reduce the electron leakage level, which is further calculated and illustrated in Fig. 4(c). Hence, the increased hole concentration and the reduced electron leakage in the quantum wells lead to the improved quantum efficiency for LED B, which is reflected by the enhanced radiative recombination rate, as shown in Fig. 4(d).

To further experimentally confirm the effectiveness of the proposed hole modulator in improving the device performance, the electroluminescence (EL) spectra for LEDs A and $\mathrm{B}$ are measured and collected by an integrating sphere attached to an Ocean Optics spectrometer (QE65000). The size of the tested LED dies is $1 \times 1 \mathrm{~mm}^{2}$ with indium bump used as the ohmic contact.

The EL spectra at the current density of 20, 30, 40, 50, and $60 \mathrm{~A} / \mathrm{cm}^{2}$ for LEDs A and B are presented in Fig. 5(a). The peak emission wavelength for LED A is $\sim 454 \mathrm{~nm}$, while it is $\sim 450 \mathrm{~nm}$ for LED B. The wavelength variation of the two LED samples is caused by the fluctuation for different growth runs. Fig. 5(a) also shows that the EL intensity for 

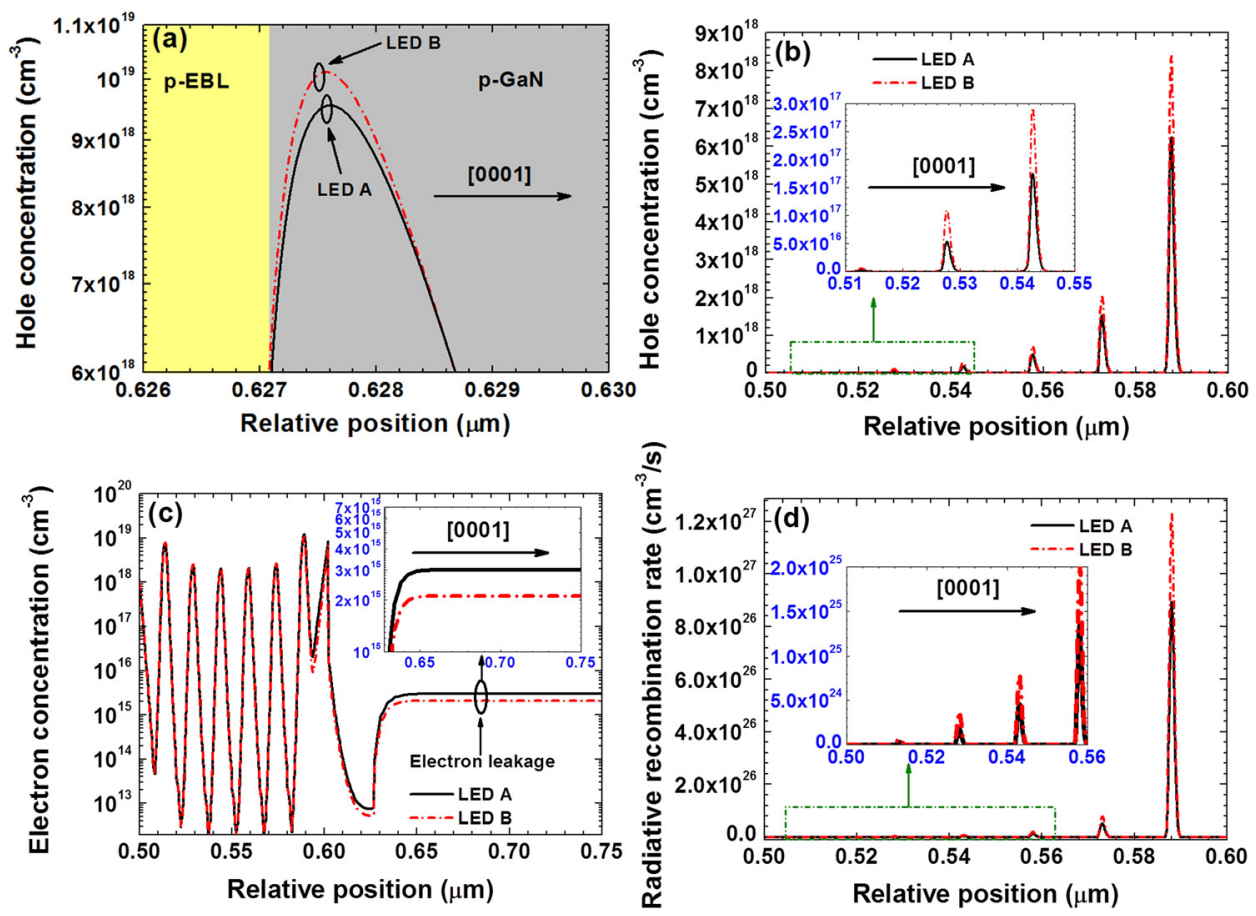

FIG. 4. Computed hole concentration profiles (a) at the interface of the p-EBL and p-GaN layer and (b) within the InGaN/GaN MQW region for LEDs $\mathrm{A}$ and $\mathrm{B}$ at $80 \mathrm{~A} / \mathrm{cm}^{2}$, respectively. (c) Computed electron profiles and (d) radiative recombination rates in the $\mathrm{InGaN} /$ $\mathrm{GaN}$ MQW region for the same devices under the same conditions.
LED B is stronger than that for LED A at all current density levels. As a result, the tested EL spectra at different current density levels allow the plotting of the optical output power density and the external quantum efficiency in terms of the injected current density for LEDs A and B. The corresponding results are shown in Fig. 5(b), from which we can see an enhanced optical output power density and external quantum efficiency from LED B compared to LED A. For example, at
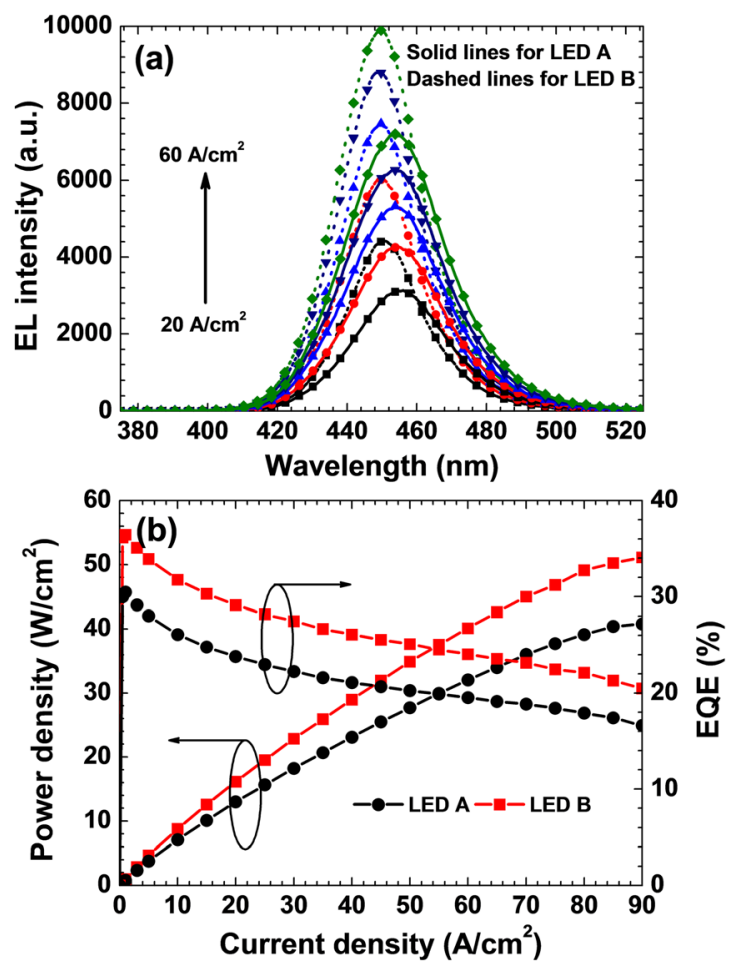

FIG. 5. Experimentally measured (a) EL spectra at 20, 30, 40, 50, and $60 \mathrm{~A} / \mathrm{cm}^{2}$, solid lines and dashed lines are for LED A and LED B, respectively, and (b) optical output power density and external quantum efficiency for LEDs A and $\mathrm{B}$, respectively. the current density of $80 \mathrm{~A} / \mathrm{cm}^{2}$, the optical output power density for LED B is increased by $25.59 \%$. The increased performance originates from the enhanced hole concentration in the p-GaN layer for LED B [refer to Figs. 3(a) and 3(b)]. The enhanced hole concentration in the p-GaN layer leads to an improved hole injection into the $\mathrm{InGaN} / \mathrm{GaN}$ MQWs for LED B.

In conclusion, we have proposed the concept of hole modulator, by which the holes generated in a remote p-type layer (e.g., the p-type doped last quantum barrier in this work) are depleted by the built-in electric field and the holes are finally stored in the $\mathrm{p}-\mathrm{GaN}$ layer. Therefore, the hole concentration of the p-GaN layer can be remarkably increased, and the effective valance band barrier height for the p-EBL is correspondingly reduced from $\sim 332 \mathrm{meV}$ to $\sim 294 \mathrm{meV}$ at $80 \mathrm{~A} / \mathrm{cm}^{2}$, which, in turn, facilitates the hole injection into the MQW active region. In addition, we have also experimentally shown the increased hole concentration in the $\mathrm{p}-\mathrm{GaN}$ layer by the capacitance-voltage measurement. The experimentally measured hole profiles are consistent with the numerically computed results, and this strongly supports the effectiveness the hole modulator in increasing hole concentration of the p-GaN layer. As a result, we have obtained the enhanced optical output power density and the external quantum efficiency in the LED sample with the hole modulator incorporated (e.g., the p-type doped last quantum barrier in this work). The p-type doped last quantum barrier is promising, and the concept of hole modulator is also very helpful in further understanding the device physics for achieving high-efficiency optoelectronic devices.

This work was supported by the National Research Foundation of Singapore under Grant Nos. NRF-CRP-62010-2, NRF-CRP11-2012-01, and NRF-RF-2009-09, and the Singapore Agency for Science, Technology and Research (A*STAR) SERC under Grant No. 1121202009. 
${ }^{1}$ S. T. Tan, X. W. Sun, H. V. Demir, and S. P. DenBaars, IEEE Photonics J. 4, 613-619 (2012).

${ }^{2}$ S. Pimputkar, J. S. Speck, S. P. DenBaars, and S. Nakamura, Nat. Photonics 3, 180 (2009).

${ }^{3}$ Z.-H. Zhang, W. Liu, S. T. Tan, Y. Ji, L. Wang, B. Zhu, Y. Zhang, S. Lu, X. Zhang, N. Hasanov, X. W. Sun, and H. V. Demir, Appl. Phys. Lett. 105, 153503 (2014).

${ }^{4}$ Z. Ju, W. Liu, Z.-H. Zhang, S. T. Tan, Y. Ji, Z. Kyaw, X. Zhang, S. Lu, Y. Zhang, and B. Zhu, Appl. Phys. Lett. 102, 243504 (2013).

${ }^{5}$ C. H. Wang, S. P. Chang, W. T. Chang, J. C. Li, Y. S. Lu, Z. Y. Li, H. C. Yang, H. C. Kuo, T. C. Lu, and S. C. Wang, Appl. Phys. Lett. 97, 181101 (2010).

${ }^{6}$ Y. Ji, Z.-H. Zhang, S. T. Tan, Z. G. Ju, Z. Kyaw, N. Hasanov, W. Liu, X. W. Sun, and H. V. Demir, Opt. Lett. 38, 202 (2013).

${ }^{7}$ Y.-K. Kuo, M.-C. Tsai, S.-H. Yen, H. Ta-Cheng, and Y.-J. Shen, IEEE J. Quantum Electron. 46, 1214 (2010).

${ }^{8}$ J. Piprek, Appl. Phys. Lett. 105, 011116 (2014).

${ }^{9}$ Z.-H. Zhang, W. Liu, Z. Ju, S. T. Tan, Y. Ji, Z. Kyaw, X. Zhang, L. Wang, X. W. Sun, and H. V. Demir, Appl. Phys. Lett. 104, 243501 (2014).

${ }^{10}$ S. Nakamura, T. Mukal, M. Senoh, and N. Iwasa, Jpn. J. Appl. Phys., Part 2 31, L139 (1992).
${ }^{11}$ A. Hiroshi, K. Masahiro, H. Kazumasa, and A. Isamu, Jpn. J. Appl. Phys., Part 2 28, L2112 (1989).

${ }^{12}$ J. Simon, V. Protasenko, C. Lian, H. Xing, and D. Jena, Science 327, 60 (2010).

${ }^{13}$ Z.-H. Zhang, S. T. Tan, Z. Kyaw, W. Liu, Y. Ji, Z. Ju, X. Zhang, X. W. Sun, and H. V. Demir, Appl. Phys. Lett. 103, 263501 (2013).

${ }^{14}$ J. H. Park, Y. D. Kim, S. Hwang, D. Meyaard, E. F. Schubert, Y. D. Han, J. W. Choi, J. Cho, and J. K. Kim, Appl. Phys. Lett. 103, 061104 (2013).

${ }^{15}$ C. S. Xia, Z. M. S. Li, and Y. Sheng, in 13th International Conference on Numerical Simulation of Optoelectronic Devices (NUSOD, 2013), p. 39.

${ }^{16}$ Z.-H. Zhang, S. T. Tan, Z. Ju, W. Liu, Y. Ji, Z. Kyaw, Y. Dikme, X. W. Sun, and H. V. Demir, J. Disp. Technol. 9, 226 (2013).

${ }^{17}$ Z.-H. Zhang, Z. G. Ju, S. T. Tan, Z. Kyaw, W. Liu, Y. Ji, X. L. Zhang, N. Hasanov, X. W. Sun, and H. V. Demir, Opt. Lett. 39, 2483 (2014).

${ }^{18}$ V. Fiorentini, F. Bernardini, and O. Ambacher, Appl. Phys. Lett. 80, 1204 (2002).

${ }^{19}$ I. Vurgaftman and J. R. Meyer, J. Appl. Phys. 94, 3675 (2003).

${ }^{20}$ D. K. Schroder, Semiconductor Material and Device Characterization, 2nd ed. (John Wiley \& Sons, 1998). 